J Ästhet Chir 2020 · 13:81

https://doi.org/10.1007/s12631-020-00232-y

(c) Springer Medizin Verlag GmbH, ein Teil von Springer Nature 2020

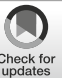

Liebe Leser des Journal für Ästhetische Chirurgie,

diese Ausgabe unseres Journals behandelt in erster Linie das Thema „energy-based devices“.

Dank an den Gastherausgeber, Herrn Dr. Gerd Kautz, und an die Autoren Prof. Gerber, Dr. Gauglitz, Dr. Kilian und Dr. Zipprich, die uns an ihrem Wissen teilhaben lassen. Herr Dr. Kautz hat in seinem Buch „Energie für die Haut“ (Springer-Verlag 2019) ja bereits mit Unterstützung zahlreicher renommierter Autoren einen sehr umfassenden aktuellen Überblick über alle Aspekte der Anwendung von Lasern und anderen „energy-based devices“ gegeben. Ich bin sehr dankbar dafür, dass wir aus diesem Buch einzelne Artikel übernehmen durften.

„Energy-based devices“ helfen durch Energieanwendungen in verschiedenen Formen, Eingriffe am Körper durchzuführen. Es handelt sich in den hier beschriebenen Verfahren in erster Linie um Laser, aber auch um andere Geräteanwendungen, wie z. B. der Radiofrequenz, mit der Behandlungen der Haut und des Unterhautgewebes erfolgen.

Seit den ersten Anwendungen der $\mathrm{CO}_{2}$-Laser vor ca. 30 Jahren in der Ästhetik können wir eine rasante Fortentwicklung vieler verschiedener Laser beobachten. Mit ganz unterschiedlichen Wellenlägen ist es nun möglich, durch Laser und IPL-Geräte gezielt Korrekturen von Pigmentstörungen, Gefäßveränderungen und Hautbildveränderungen durchzuführen sowohl in der Ästhetik, aber vielleicht $\mathrm{zu}$ einem noch größeren Anteil kurativ. Bei den anderen „energy-based devices" handelt es sich um Geräte, die mit Ultraschall, Radiofrequenz, reiner Wärme oder Kälte die

\title{
Frank Muggenthaler
}

Muggenthaler Ästhetik - Klinik für Plastische Chirurgie und Ästhetische Medizin GmbH, Gutach, Deutschland

\section{„Energy-based devices“}

verschiedensten Effekte bewirken, v. a. zur Gewebestraffung oder -formung.

Während die Behandlungsoptionen immer umfangreicher werden und die Auswahl der zur Verfügung stehenden Geräte immer unübersichtlicher zu sein scheint, können wir auch einen Trend beobachten, der Grund zur Besorgnis auslösen sollte. Auch Nicht-Mediziner gelangen in den Besitz solcher Geräte und bieten die vermeintlich einfachen Behandlungen an.

Vor diesem Hintergrund bin ich den Autoren dankbar für ihre Darstellung einzelner sehr wichtiger Anwendungen, aber v. a. auch der möglichen Komplikationen. Eindrücklich wird damit gezeigt, wie anspruchsvoll Laseranwendungen sind, welches differenzierte Wissen um die technischen und physikalischen Grundlagen der Behandlungen vorausgesetzt werden muss und wie wichtig es eben auch ist, dass ausschließlich gut ausgebildete Anwender diese Methoden anwenden und die Geräte nicht in die Hände von Nicht-Medizinern gelangen sollten. Jedem verantwortungsbewussten Kollegen wird spätestens bei der Lektüre der Artikel in diesem Journal bewusst, wie viel Wissen erforderlich ist, um die Techniken effektiv und sicher durchzuführen, und welchen Respekt die geübten Anwender daher verdienen.

Vor diesem Hintergrund ist es auch nachvollziehbar, dass die Novelle der Strahlenschutzverordnung den Gebrauch aller Geräte, welche mit Energiewellen unterschiedlicher Frequenzen arbeiten, neu regelt.

Es ist mir aber auch wichtig, auf einen Punkt hinzuweisen, der nicht so sehr das rein Medizinische betrifft, sondern unsere Gesundheitspolitik:

Auch wenn Laser für viele Belange der Ästhetik erfolgreich angewandt wer- den, liegt ihr Haupteinsatzbereich wahrscheinlich in der kurativen Medizin. Es ist wirklich ein Ärgernis, dass eine wirtschaftliche Anwendung der Geräte eigentlich nur bei privat versicherten $\mathrm{Pa}$ tienten möglich ist. Auch wenn dieser Missstand ja in vielen Bereichen unseres Gesundheitssystems besteht, ist es dennoch wichtig, darauf hinzuweisen.

Ich wünsche Ihnen viel Freude und möglichst viele wertvolle neue Erkenntnisse beim Lesen dieses Journals.

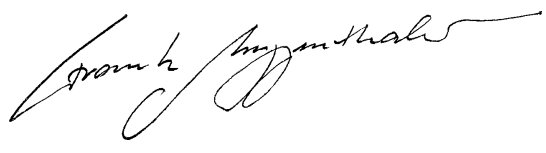

Dr. Dr. Frank Muggenthaler

Schriftleiter der JÄC und Präsident der GÄCD

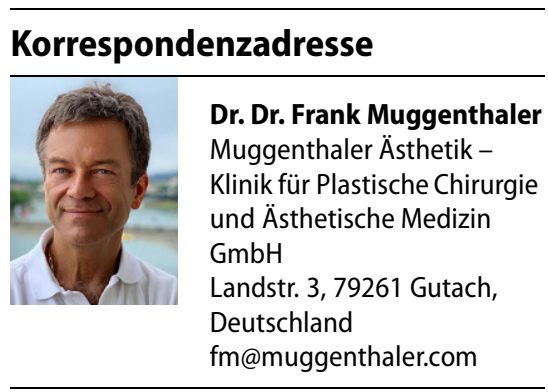

Interessenkonflikt. F. Muggenthaler gibt an, dass kein Interessenkonflikt besteht. 